\title{
Linx
}

Revue des linguistes de l'université Paris X Nanterre

9 | 1997

Émile Benveniste. Vingt ans après

\section{La langue : clivage, nouages, capitonnage}

\section{Marc Derycke}

\section{(2) OpenEdition}

\section{Journals}

Édition électronique

URL : http://journals.openedition.org/linx/1066

DOI : 10.4000/linx.1066

ISSN : 2118-9692

\section{Éditeur}

Presses universitaires de Paris Nanterre

\section{Édition imprimée}

Date de publication : 1 avril 1997

Pagination : 271-286

ISSN : 0246-8743

\section{Référence électronique}

Marc Derycke, «La langue : clivage, nouages, capitonnage », Linx [En ligne], 9 | 1997, mis en ligne le 09 juillet 2012, consulté le 30 avril 2019. URL : http://journals.openedition.org/linx/1066 ; DOI : 10.4000/ linx.1066 


\title{
La langue : clivage, nouages, capitonnage
}

\author{
Marc Derycke
}

$\mathrm{J}$

e prendrai ici pour objet la conception de la langue que défend E. Benveniste et la signifiance de ses concepts à partir de la séparation entre sémiotique et sémantique ${ }^{1}$.

Son oeuvre constitue notre donné sémiotique. Il est certes possible de calculer les bornes et l'erre des références potentielles qu'induisent ses significations à condition d'en forclore le sujet. Mais peut-on discerner la référence hic et nunc soit "20 ans après", référence actuelle une et non pas multiple, particulière et non pas "générique", car désignée par la langue "en emploi", celle du texte $l u$ ?

La lecture est effet d'un sujet épinglé par la langue, aussi effacé soit-il en sciences, et qui se manifeste parfois non sans surprise par sa visée, par une activité sémantique visant à introduire un principe d'unité dans l'hétérogénéité textuelle : ce principe selon sa nature est susceptible de révéler des cohérences variées et de degrés divers, ce dont d'autres lectures témoignent ${ }^{2}$. Mettre en évidence une cohérence implique donc forçage d'une consistance par l'autre opérant nécessairement sur ce qui semble être des parties, en ce qu'elles servent à une construction : ce ne sont pas en effet les mêmes segments qui y font "pavé dans la mare", on n'en recueille donc pas la même écume. Surtout cette "coopération textuelle" du lecteur (Eco) introduit une extériorité par quoi il n'existe pas d'immanentisme absolu : le découpage textuel et la hiérarchisation des segments est commandée par les objectifs et les références particulières de l'interprète ; la lecture est aussi greffe qui s'ignore.

La mienne sera greffe revendiquée, elle tentera d'expliciter des points impliqués par le système conceptuel ébauché par Benveniste, points obscurs

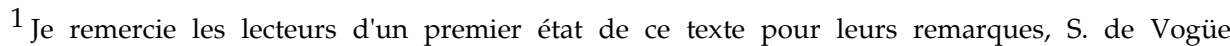
notamment avec qui quelques divergences subsistent.

2 D'où les convergences et / ou les écarts avec Tamba-Mecz (1984), Culioli et Hagège (id.), Normand (1986 et 1989), ainsi que dans $\operatorname{Lin} x \mathrm{n}^{\circ}$ 26, 1992, Normand, de Vogüe, Montaut.
} 


\section{Marc Derycke}

signalés par ses commentateurs. Pour moi, référence faite à la théorie lacanienne du signifiant, il s'agira de problématiser la place du sémantique par rapport à l'autonomie de la langue comme système, question réactualisée par le cognitivisme et la tentation d'un nouvel amalgame entre langue et pensée en didactique selon quoi la première serait encore instrument de la seconde. Je supposerai donc que, dégagée de la civilité de l'hommage, cette lecture peu naïve mais si peu rusée, quêtant des lumières auprès d'un "Benveniste" pour un bel "aujourd'hui", produira un effet de vérité sur l'oeuvre, "20 ans après".

\section{Clivage de la langue}

Le clivage entre sémiotique et sémantique a déjà été présenté, citations à l'appui ${ }^{3}$. Rappelons donc qu'"il n'y a pas transition" du "signe" à la "phrase", mais qu'"un hiatus les sépare" par quoi "la langue comporte deux domaines distincts dont chacun demande son propre appareil conceptuel" (II : 65, 1969, je souligne); ceci transparaît déjà en 1962 : des mots à la phrase, la "progression semble linéaire", mais "une situation toute différente va se présenter ici" (I : 124) : chacun est à l'autre sans mesure commune dans un non-rapport.

Le sémiotique est caractérisé par le système. Son unité est le signe, il a pour fonction de signifier (II : 64), son identité tient à sa valeur, soit l'ensemble des relations qu'il entretient avec la totalité. Il lui suffit d'être RECONNU, dès lors ce qu'il "signifie n'a pas à être défini" (II : 222, 1966). De cela on déduira que, rapporté à sa capacité supposée de désigner le référent, opération toujours singulière, le signifié est indéterminé car de "valeur générique et conceptuelle" (II : 223) : il a pour fonction constitutive de distinguer le signe d'un flatus vocis. Le signe vaut donc pour un quasi-signifiant, dès lors trouvent place dans son ordre tous les termes de la langue, dont les marques du discours et le nom propre; son sens sémiotique lui vient de sa "fonction intégrative" (I : 125), soit celle d'intégrer un niveau supérieur.

Le sémantique doit être COMPRIS (II : 65), il a pour fonction de communiquer. Ce mode a à charge d'établir "quel est ce sens" ce qui mène à "dire quel est le référend" (I : 127 - 128). La référence relève de la fonction prédicative de la phrase. Cette dernière "est l'expression sémantique par excellence" (II : 224), son statut conceptuel est soumis entièrement à sa capacité de désignation. La conception d'E.B. lue ici est parallèle à celle de la sémantique en logique si on en retranche le jugement sur la vérité ; ainsi peut-on entendre la phrase comme l'expression qui subsume un concept "unique" (l'idée") sous lequel tombe un objet, le référent.

\footnotetext{
${ }^{3}$ Cf. Derycke (1995) et note précédente. Les références signaleront le tome des PLG suivi de l'année de publication de l'article.
} 
Le sens, présent dans les deux modes de "signifiance", est cause du "hiatus"4 qui les sépare de par ses deux acceptions : en sémiotique il tient à la fonction intégrative du signe mais aussi à sa valeur par quoi se guide sa plurivocité, alors qu'en sémantique, il dépend de la relation de la phrase au référent, qui elle vise l'univocité ${ }^{5}$. Dès lors, ce qui est en cause, c'est la relation du signifiant à un signifié déterminé, car "particulier ou occasionnel" (II : 223) .

Conséquence du clivage de la langue, les mêmes matériaux vont fonctionner et être décrits par deux "appareils conceptuels différents". Cette identité phénoménale des unités de langue risque à tout moment d'effacer sa division, c'est pourquoi il est nécessaire d'en présenter le critère qui me semble le plus opératoire : la transposition.

On peut transposer le sémantisme d'une langue dans celui d'une autre "salva veritate" ; c'est la possibilité de la traduction; mais on ne peut pas transposer le sémiotisme d'une langue dans celui d'une autre, c'est l'impossibilité de la traduction. (II : 228, 1966)

Frege utilise ce critère en logique pour différencier le Sinn de la Bedeutung et délimite incidemment le sémiotique : à "dénotation" constante, la substitution d'unités d'une même proposition fait varier leur "sens" (Frege, 1971: 129, 136, et passim). A ce point de disjonction épistémologique et méthodologique entre sémiotique et sémantique, se pose la question de savoir quel objet va se donner Benveniste pour en entreprendre la description, sera-ce la langue selon le mode sémantique (sans sémiotique), ou plus conformément à sa discipline, selon le sémiotique (sans sémantique), à l'inverse du geste fondateur en logique ${ }^{7}$ ?

\footnotetext{
${ }^{4}$ Ce qui renvoie aussi à interruption, espace, interstice, lacune, le hiatus marque fortement une absence de médiation permettant une relation homogène ou symétrique entre les deux "domaines", appelés aussi "modes", et que j'identifie à des "consistances".

5 Sous réserve d'inventaire, univocité n'apparaît pas chez E.B., mais tout l'indique ici, par ailleurs cette propriété a peu été abordée à l'époque (cf. Arrivé, 1994 : 190).

${ }^{6}$ Cette incompatibilité, loin d'être nouvelle, se déduit de Saussure que cite Benveniste : "C'est une grande illusion de considérer un terme comme l'union d'un certain son avec un certain concept. Le définir ainsi, ce serait l'isoler du système (...) ; ce serait croire qu'on peut commencer par les termes et construire le système en en faisant la somme" (I : 92, 1962, je souligne).

7 "On a tendance (...) à croire que diverses expressions d'un langage ne sont jamais parfaitement équivalentes et qu'un mot ne peut jamais être rendu avec exactitude dans un autre langage. (...) j'insisterai seulement sur le fait que l'on peut trouver un élément commun en diverses expressions, ce que j'appelle le sens et, dans le cas des propositions, la pensée. (...) On peut exprimer différemment le même sens, (...) la différence n'est pas alors celle du sens mais une différence de manière de voir, d'insistance, de coloration, que la logique ne prend pas en compte." (Frege, 1971 : 131, note 1 , je souligne). La condition de description du sémantique repose alors sur la suspension du sémiotique de la langue, c'est-à-dire du matériau qui manifeste la "diversité" d' "expression" soit d'un même "sens", soit d'une même "dénotation" au travers de "sens" différents.
} 


\section{Marc Derycke}

Je soutiendrai que le défi de Benveniste est de maintenir comme objet les deux modes incompatibles, les tenant raboutés par une série de nouages par quoi "cela tient", ce dont la tradition scientifique s'accommode mal. En effet je soutiens la thèse que pour Benveniste la langue est une mais aussi double tout $\grave{a}$ la fois, c'est-à-dire composée de deux consistances distinctes qui sont et sans rapport l'une avec l'autre et inséparables totalement car nécessaires l'une à l'autre pour leur constitution même ${ }^{8}$ : aucune ne peut se déprendre de son double.

\section{Nouages}

Le premier nouage, essentiel, est réalisé par l'exigence à laquelle E.B. fait droit: le sémantique ne peut être exclu du sémiotique, il est nécessaire à la constitution de ses unités. En effet leur délimitation est conditionnée par le sens, au travers de la fonction intégrative : "plutôt que de biaiser avec le "sens", et d'imaginer des procédés compliqués - et inopérants - pour le laisser hors de jeu en retenant seulement les traits formels, mieux vaut reconnaître franchement qu'il est une condition indispensable à l'analyse linguistique. (...) Ce niveau (du sens, n.d.r.) n'est pas quelque chose d'extérieur à l'analyse, il est dans l'analyse" (I : 122). Cette position ne sera jamais mise en cause par la suite. Mais interrogeons ce "sens" introduit "dans l'analyse". Il est à considérer selon les deux consistances : $1^{\circ}$ il est sémiotique par l'opération formelle d'intégration, l'autonomie de l'appareil conceptuel est alors préservée, $2^{\circ}$ mais il est aussi sémantique et condition même du sens sémiotique, c'est en cela qu'il y a nouage avec le double exclu et incompatible : la fonction intégrative anticipe l'autre consistance car elle est suspendue à la capacité référentielle que possède la fonction prédicative de la phrase?.

Le nouage en retour s'effectue dans le discours, c'est-à-dire avec "la langue en emploi et en action" (II : 224). Cette fois le sémiotique, bien que dominé, est indéfectiblement lié au sémantique qui ne peut s'en affranchir : le matériau de la phrase est sa condition même ${ }^{10}$. Le bouclage s'achève au plan lexical quand le sens (appelé "notion") est structuré par le "rapport de signification" qui dépend du sémiotique. Ainsi ces deux termes associés à une notion semblable ne sont pas permutables, civitas ne peut se substituer à polis

\footnotetext{
${ }^{8}$ De plus l'une pénètre l'autre et vice versa (cf. de Vogüe, ici même).

${ }^{9} \mathrm{La}$ "fonction propositionnelle" qui réalise l'intégration ("Niveaux...") recouvre en fait deux fonctions distinctes structurant respectivement chaque consistance : $1^{\circ}$ la fonction propositionnelle de tel signe compte non tenu du sens de la phrase - c'est-à-dire hors prédication induisant une référence, $2^{\circ}$ la fonction propositionnelle entendue comme fonction prédicative permettant la désignation d'où se déduit le sens des mots ; la première fonde le sens des unités en sémiotique, la seconde fonde le sémantique.

10 C'est en quoi la langue se différencie d'autres langages, tels la peinture et la musique, constitués d'une "sémantique (...) sans sémiotique" (II : 65, je souligne).
} 
parce qu'ils appartiennent à des systèmes en rapport inverse : civitas / civis // polis / politès (II : 274 et sq., 1970), ce rapport engage une différence notionnelle.

Ces nouages apparaissent dans le texte par des tours d'écriture qui ne sont donc pas fortuits.

En des points stratégiques de la théorie, textes "canoniques" ou "d'arrêt" (Normand, 1986: 195), E.B. tient ensemble deux propriétés disjointes, incompatibles ou en exclusion réciproque. Nouage signifiera ici maintien des deux modes en un point local de l'objet langue soit in praesentia: et l'un et l'autre concept - avec ou sans rapport de domination, soit in absentia : pas l'un sans l'autre.

Conjonction : et ... et ...

(1) "... double nature profondément paradoxale du langage, à la fois immanente ... et transcendante" (II : 95, 1968)

(2') Il y a pour la langue deux manière d'être langue dans le sens et dans la forme. (II : 224, 1966)

(2') "sens et désignation (...) distincts mais associés au niveau de la phrase." (I : 128, 1962)

(3) "Il (Saussure) montre admirablement qu'on peut parler à la fois de l'immutabilité et de la mutabilité du signe (...) parce que (il est) arbitraire ..." (I : 53, 1939)

(4) la relation en même temps se modifie et reste immuable (...) entre le signe et l'objet (ibid.)

(5) synchronie d'un système toujours menacé, toujours restauré (id. : 54, je souligne de (1) à (5))

Dans (1) et (2) les termes sont en relation de contrariété, en outre dans (2) la langue est montrée comme une et double à la fois. Là l'opposition sémiotique / sémantique est désignée alors qu'elle ne l'était pas encore en 1939, néanmoins les exemples (3) à (5) la désignent indirectement marquant la contradiction entre mutabilité du signe soumis à l'usage (activité - sémantique - de discours) et immutabilité du signe dans le système, l'"arbitraire" permettant la bascule de l'un à l'autre. Partout les propriétés de la langue sont désignées par des termes marquant une opposition forte et simultanée usant même de l'oxymore. En somme

$1^{\circ}$ la langue fait échec au principe de non contradiction,

$2^{\circ}$ E.B. intègre cette contradiction à l'objet de la discipline et la serre, arrimant les bords ; en conséquence 


\section{Marc Derycke}

a) serrage ${ }^{11}$ des deux consistances n'est pas synthèse : un reste est isolé qui n'est pas rien, il est même constitutif : c'est le sémiotique en ce qu'il n'est pas traductible (cf. le critère évoqué supra), il excède ainsi le sémantique,

b) avec le maintien de termes opposés par un tour d'écriture, E.B. instaure entre eux une relation instable. Ainsi les deux bords, identifiés au niveau de l'objet, sont noués par figure dans le métalangage, ceci à l'encontre de la démarche classique selon quoi, nanti de concepts univoques, il faudrait établir une séparation stricte, un mode sans l'autre, pour en décrire ensuite les propriétés, ce qui laisserait échapper le double hétérogène qui constitue de l'intérieur la langue.

\section{Disjonction : pas ... sans}

Les concepts qui s'excluent mutuellement de par leur appartenance aux deux domaines sont néanmoins opérationnellement solidaires en ce qu'ils sont liés dans leur constitution par une double présupposition (cf. supra les deux nouages) : pas l'un sans l'autre, même si chaque domaine "demande son propre appareil conceptuel". C'est notamment ce que montre sans cesse E.B. à propos de la forme et du sens dans "Niveaux de l'analyse linguistique".

Considérant les couples qui font système pour décrire le double fonctionnement de la langue, il suit qu'il n'est pas de forme qui puisse être constituée sans appel au sens, pas de paradigme sans recours au syntagme, pas de constituant sans intégrant (anticipation sur la fonction prédicative), etc. : rappelons-le, pour identifier une forme, on ne peut "biaiser avec le sens", dès lors le reste suit, on ne peut construire un paradigme indépendamment des syntagmes où il est intégrable. Asserter un de ces concepts, c'est simultanément envisager sa suppression et y loger l'opposé auquel il est corrélé et qui le structure au plus intime parce qu'il en est la condition de possibilité. Ainsi caractériser le sémiotique par la relation paradigmatique (cf. (6)), "relation de l'élément avec les autres éléments mutuellement substituables" (I : 120), c'est aussi indiquer en creux le syntagmatique. Reste à savoir si les oppositions virtuellement équipollentes, le sont toujours dans leurs actualisations.

\section{Association, domination}

En fait le clivage de la langue rend impossible la transformation réciproque. Par exemple la fonction intégrative n'est pas la réciproque de l'analyse en constituants bien qu'elles se présupposent mutuellement. Cet effet n'est pas toujours lu chez E.B. Ainsi

\footnotetext{
11 Nouage désigne la topologie des noeuds (cf. Derycke 1990, VIe partie), quant au "serrage", introduit précocement par Lacan à propos du capitonnage (Lacan, $1981: 316)$, il désigne dans le dernier état de sa théorie le blocage du triskel (concours de trois consistances) qui rend compte du trait unaire ; ainsi le serrage "coince", identique au trait articulant la différence.
} 
(6) "les signes se disposent toujours et seulement en relation paradigmatique." (II : 223, 1966)

(7) "le sens (sémantique) s'accomplit dans et par une forme spécifique, celle du syntagme. (...) Tout (= choix, agencement des mots) est dominé par la condition du syntagme," (II : 225, je souligne)

(8) "Les relations paradigmatiques comptent moins, en tant qu'il s'agit du mot, par rapport à la phrase." (I : 124, 1962, idem)

Autrement dit, si le sémiotique exclut positivement le syntagmatique en tant que fonction prédicative actualisée - interprétation qui me semble tenable dès l'article de 1962 (cf. citation (9) et note 21), par contre le paradigmatique, soit le mode sémiotique auquel appartiennent les unités soustraites au syntagme, demeure présent mais dominé en sémantique, position stable à quelques années de distance (cf. aussi citations (10) à (12)).

\section{Le concept comme figure}

Les principaux concepts descriptifs semblent sémantiquement duplices, ceci n'a pas échappé à Tamba, à Culioli qui reproche un "brouillage". Ainsi, en est-il de langue, mot / signe, phrase $^{12}$.

Ainsi "langue" réalise souvent une sorte de catachrèse pouvant désigner la langue et comme système et / ou comme discours; par ailleurs elle peut occuper les deux places dans $\mathrm{S} \rightarrow \mathrm{L}$ : et comme système particulier en position d'interprété (en S), et, dans son fonctionnement discursif, comme interprétant (en L), par quoi la langue est capable de se prendre elle-même pour objet (II : $54,1969)$ et de réaliser sa "conversion (...) en discours" (II : 81, 1970).

"Signe", unité du sémiotique, et "mot", du sémantique, désignent un même élément pour distinguer son mode de fonctionnement. Leur usage peut faire l'objet de confusions (cf. Tamba, 1984 : 190-192).

(9) "Nous pouvons (...) classer les signes comme une seule espèce, qui coïncidera pratiquement avec le mot." (I : 123)

"Phrase" est le concept décisif qui manifeste empiriquement la différence entre sémiotique et sémantique. Il n'existe pas de sémiotique de la phrase, soutien E.B. contre la proposition de Ricoeur (II : 237) ; son statut semble donc non ambigu car suspendu à sa fonction sémantique, la référence, par quoi elle

\footnotetext{
12 Le problème intéresse E.B., par delà sa réfutation d'Abel via Freud (1956) : "C'est par suite de leur coaptation que les mots contractent des valeurs que en eux-mêmes ils ne possédaient pas et qui sont même contradictoires avec celles qu'ils possèdent par ailleurs. On voit s'allier des concepts logiquement opposés et qui même se renforcent en se conjoignant." (II : 227, 1966). Ce qu'il observe ici chez des unités prises dans des relations syntagmatiques au niveau du langage objet, on le voit paradoxalement fonctionner au niveau des unités du métalangage qu'il élabore, le contexte étant cette fois la théorie descriptive elle-même.
} 


\section{Marc Derycke}

subsume "l'idée". Cependant l'arrière-plan sémiotique de la phrase est perceptible et démarque sa définition de celle de la proposition en logique. En effet la dénotation de cette dernière se réduit sans reste à la composition des parties, lesquelles sont substituables "salva veritate" (cf. les principes de compositionnalité et de substituabilité au sein de la proposition chez Frege). Néanmoins E.B., contre tout formalisme, maintient cet arrière-plan à plusieurs reprises :

(10) "(la phrase) porte à la fois sens et référence : sens parce que elle est informée de signification ..." (I : 130)

(11) "Une phrase constitue un tout, qui ne se réduit pas à la somme de ses parties ;" (I : 123, 1962)

(12) "le sens d'une phrase est autre chose que le sens des mots qui la composent" (II : 226, 1966, je souligne)

Dans (10) "signification" renvoie au sens en sémiotique, non à celui qui résulte de "l'idée" ; de même (11) signale un reste par rapport à la réduction du sémiotique par le logicien, le sens est excès et fonds d'où le sémantique surgit en l'occultant. La formulation qui lui succède (12), concordante certes avec une distinction affinée entre "signe" et "mot", fait apparaître encore ce reste: le présent du verbe clive le sens contradictoirement réalisé en même temps dans la phrase d'une part et dans ses parties de l'autre ${ }^{13}$. Ceci est cohérent avec l'article puisqu'y est mis en évidence la non symétrie entre paradigme et syntagme (cf. citations (6) et (7), supra) : le premier exclut le second mais dans la phrase le second est associé au premier et le domine, c'est pourquoi il n'est pas impossible que telle virtualité du paradigme puisse inopinément surgir, remaniant la référence (cf. infra, le Witz) ${ }^{14}$. En 1969 ce fonds sémiotique est rappelé avant d'en détacher, incompatible, le sens propre à la phrase : "le message ne se réduit pas à une succession d'unités à identifier séparément" (II : 64, 1969), ce reste est marqué par le même lexème qu'en 1962 : réduire (cf. (11)). Cette duplicité du sens dans la phrase constitue la pierre de touche qui démarque la sémantique de la langue selon E.B. de celle du logicien.

\footnotetext{
13 On objectera que les formulations (10) et (11) expriment un lieu commun. Elles ne le sont plus dès qu'on les considère dans l'économie conceptuelle nécessitée par le hiatus entre sémiotique et sémantique qui, lui, semble loin d'être une banalité. Une proposition du type "le sens de la phrase diffère du sens que possèdent ses composants dans leur paradigme respectif hors leur présente actualisation" aurait levé l'ambiguïté du texte. Par ailleurs "somme" est à prendre pour une opération quelconque de composition, ce que (12) vérifie.

14 Par exemple : "il vaudrait mieux que je quitte l'enseignement ... même si je dois commencer par le bas". Introduit par le (et non par en), "bas" pourra désigner le bas féminin sous certaines conditions non exemptes de malice.
} 
La langue : clivage, nouages, capitonnage

\section{Le métalangage et le Witz}

E.B. est intransigeant sur le respect du principe de non contradiction (cf. I : 110, 1952-53) : une description doit être "consistante et compréhensive" (id. : 111). Mais, référant à la typologie de Sapir, lorsque se pose l'alternative entre le respect des langues comme objet menant aux répartitions "indécises", aux "nuancements gradués", et celui du tranchant apparent de la cohérence formelle, E.B. prend le parti de Sapir ${ }^{15}$. E.B. ne craint donc pas d'affronter les limites de la théorie quand elle est imposée par son objet. Ainsi écrit-il à propos de Saussure et de l'arbitraire du signe ${ }^{16}$ : "C'est peut-être le meilleur témoignage de la fécondité d'une doctrine que d'engendrer la contradiction qui la promeut" (I : 55, 1939). La formule, à cette époque, est aussi programmatique. En effet, loin de se détourner de l'arbitraire, E.B. cherchera à intégrer le dehors qui contraint la langue, élaborant un métalangage qu'il ajustera en même temps qu'est théorisé le clivage. C'est pourquoi il maintient raboutés les deux consistances au risque de trivialiser les concepts. Leur ambiguïté apparente est aussi mise en scène de la division, ce que souligne la récurrence des connexions quasi figuratives du type "à la fois", "en même temps", etc. (cf. (0) à (5)).

En maintenant dans la phrase un reste sémiotique qui excède le calcul de la référence (cf. supra, (11), (12)) et qui, simultanément, en est exclu pour cette raison, E.B. soutient une conception de la langue difficile et exigeante puisqu'elle semble frôler la contradiction. Or, loin d'être inconsistante, cette conception fonde la langue comme gîte du sujet clivé, réserve de sa liberté. C'est sur elle que s'appuie la description par Freud du mot d'esprit :

$1^{\circ}$ le Witz n'est possible que par la conjonction de deux conditions contradictoires a) présence du mot, "pont verbal", tel qu'il "habille deux pensées" (c'est la fonction SIGNIFIER du sémiotique et ses possibilités associatives (II : 64)), b) réalisation de la fonction prédicative, selon E.B., celle de COMMUNIQUER, "façade" qui capte l'attention du sujet vers la référence attendue, par quoi le sens du Witz survient et sur-prend : le pas de sens (Lacan, Séminaire V), l'exclu du sémantique, est à entendre aussi comme passage du sens ;

\footnotetext{
15 Il le cite : "Il est moins important de loger chaque langue dans une petite case bien nette que d'avoir développé une méthode souple qui nous permet de la situer, de deux ou trois points de vue indépendants, par rapport à une autre langue" (id. 114). Ceci peut être transposé à la langue et aux propriétés requises du métalangage confronté à son clivage.

16 Arbitraire qu'il déplace vers le référent, ce dont son oeuvre prendra la mesure : il y a rapport "nécessaire" du signifiant au signifié, il relève du sémiotique, alors que le sémantique est effet du rapport "arbitraire" du signe au référent, d'où l'"hiatus".
} 


\section{Marc Derycke}

$2^{\circ}$ la "transposition" du mot d'esprit, (Freud : 140 et 68-69) permet d'isoler les deux consistances de la langue selon le même critère qu'E.B. : la traduction supprime le plaisiri ${ }^{17}$.

Pensé par le truchement de concepts quasi freudiens (car de "sens opposés"18), l' "hiatus" fournit la base théorique qui permet de rendre compte et des conditions de manipulation et du langage qu'investit une subjectivité qui excède celle à quoi réfère E.B. par les marques énonciatives. Le sujet de l'énonciation est absent de la théorie E.B. (Normand, 1986 : 202), une place y est donc en réserve pour le concevoir en rapport à la nature de $s a$ langue, celle dont il est serf et qu'il ne cesse de s'approprier. Clivé, il joue sa partie dans l'interstice entre sémiotique et sémantique, possiblement en tous ses lieux. Reste à rendre compte du principe d'ordre qui scande la constitution du sens et de la signification et faire surgir ainsi un autre niveau de cohérence.

\section{Capitonnage}

E.B. introduit les traces d'un ordre dans les avènements sémanticosémiotiques.

\section{Premier ordre}

(13) "le "sens" de la phrase est dans la totalité de l'idée perçue par une compréhension globale ;" (II : 228)

(14) "une phrase constitue un tout (...) le sens inhérent à ce tout est réparti sur l'ensemble des constituants." (I : 123)

Il y a donc deux temps logiques: dans le premier, la phrase surgit comme un tout de par sa fonction de prédication (cf. (11) et I : 129), dans le second se déduit de ce tout le sens de ses constituants, les "mots", qui "en effectue(nt) la signification". Reste à identifier l'origine raturée par E.B., soit le matériau préexistant d'où émerge la phrase comme unité de désignation dans l'acte de discours. Pour reconstruire ce temps zéro de l'origine, je référerai à Lacan (séminaire III), qui permet ici d'opérer une jonction :

(15) "La phrase n'existe qu'achevée, (cf.(16)). Une unité signifiante (= le "mot" selon E.B., n.d.r.) suppose une certaine boucle bouclée qui en situe les divers éléments." (Lacan, 1981 : 297-298)

(16) "et son sens lui vient après coup" (ibid.)

\footnotetext{
17 A propos du sens et de la signification dans le Witz, cf. Derycke 1990, Ve partie.

18 Référant aux opérations présidant à la relation d'intégrant et de constituant, E.B. écrit : "Voilà deux opérations complémentaires de sens opposé" (I : 125). Plus généralement les concepts appartenant chacun à un appareil se présupposent mutuellement en s'excluant de manière non réciproque et forment des couples (v. supra).
} 
On saisit l'identité de (15) avec (13) et (14), ce qui rend d'autant plus aisée la greffe de l"après coup" du sens (16) dans la théorie benvenistienne, après coup indispensable pour penser le surgissement de la désignation là où il n'existe rien qui permette de la calculer anticipativement par quelque relation entre sémiotique et sémantique ${ }^{19}$. L'après coup prend la mesure du nonrapport entre ces "deux manières d'être langue", non-rapport fondé sur l'arbitraire radical entre le signe et la "chose" (1939). Mais poursuivons avec Lacan: le sens du message, "aucune accumulation, aucune superposition, aucune somme de significations ne peut suffire à (le) justifier" (id. : 303) ; le parallèle est complet avec E.B. Ici encore Lacan explicite ce qui est lacunaire dans la théorie d'E.B. : il y a "transmutation" entre les éléments et la phrase achevée. Avec ces indications, il est possible de reconstruire le mode de fonctionnement préalable à l'avènement de la phrase qui, bien que "réduit", y insiste néanmoins: cette non-phrase est suite d'éléments actualisés de pur sémiotique - c'est-à-dire de signes reconnus, distingués d'un flatus vocis - dont la "somme" n'est pas identique au "tout", "parties" disjointes de la phrase à venir, futurs "mots" encore juxtaposés qui presque la "composent" (cf. (11) et (12)) pour se figer bientôt en un précipité de désignation, mais entretiennent entre eux encore des relations paradigmatiques, supports des associations. Je dénomme amorce ce mode sémiotique qui va être "transmuté" dans la phrase. Le saut qualitatif de l'amorce (sémiotique) à la phrase (sémantique) se réalise par ce que Lacan a appelé "le point de capiton"20. L'Autre (A) est le système des signes tel qu'E.B. le définit. La constitution de la phrase (P) commence avec l'amorce qui, anticipant le sens, est configurée par prélèvement dans le sémiotique (fig.I). La scansion va conjoindre simultanément en un "point noeud", à un certain "moment", un "lieu", le paradigme (flèche verticale passant par A), et la temporalité du syntagme (flèche horizontale) par quoi l'amorce se métamorphose en phrase, "totalité" (E.B.) dotée d'une "signification comme produit fini" (s(A), fig.II, identique à $\mathrm{P})$, "point où viennent se nouer le signifié

\footnotetext{
19 E.B. est particulièrement clair à ce sujet et prend à rebours les théories de la lecture, fondées sur l'anticipation et la vérification d'indices, qui lui sont postérieures : le sens "implique référence", état ou situation que "nous ne pouvons jamais ni prévoir ni deviner" (II : 227), etc., la désignation du message n'est pas obtenue par la "somme" progressive de ses unités (cf. (11) et II : 64).

20 "Ce point de capiton, trouvez-en la fonction diachronique dans la phrase, pour autant qu'elle ne boucle sa signification qu'avec son dernier terme, chaque terme étant anticipé dans la construction des autres, et inversement scellant leur sens par son effet rétroactif. (Voici) la fonction des deux points de croisement dans ce graphe primaire. L'un, connoté A, est le lieu du trésor du signifiant, ce qui ne veut pas dire du code, car ce n'est pas que s'y conserve la correspondance univoque d'un signe à quelque chose, mais que le signifiant ne se constitue que d'un rassemblement synchronique et dénombrable où aucun ne se soutient que du principe de son opposition à chacun des autres. L'autre, connoté $s(\mathrm{~A})$, est ce qu'on peut appeler la ponctuation où la signification se constitue comme produit fini. Observons la dissymétrie de l'un qui est un lieu (place plutôt qu'espace) à l'autre qui est un moment (scansion plutôt que durée)." (Lacan, $1966: 805-806$, v. fig. II).
} 


\section{Marc Derycke}

et le signifiant, entre la masse toujours flottante des significations" (ibid., je souligne), ou encore "point de serrage dans un faisceau de significations" (id. : 316).

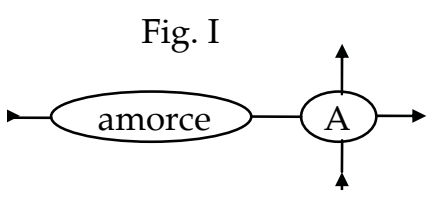

Fig. II

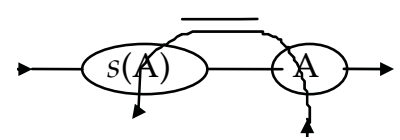

Le premier temps, selon E.B., est sémantique, il valide le temps zéro, sémiotique, ici reconstruit.

\section{Second ordre}

(17) "par comparaison et analyse diachronique (il s'agit) de faire apparaître une signification là où, au départ, nous n'avions qu'une désignation" (E.B., 1969 I : 11).

De là suit, $1^{\circ}$ que la désignation, qui relève de la langue en emploi et donc de la phrase, précède l'établissement de la signification, $2^{\circ}$ que la signification n'est pas une désignation; je soutiendrai qu'elle est une transformation de désignations.

Qu'il s'agisse de "problèmes sémantiques de la reconstruction" ou de l'établissement du vocabulaire des institutions indo-européennes, E.B. est confronté à un apparent paradoxe lié au clivage de la langue.

Soit une position théorique, s'appuyer sur le "seul principe" selon lequel "le "sens" d'une forme linguistique se définit par la totalité de ses emplois" (I : 289-290). Or on sait quelle distance Benveniste prend avec la totalité quand elle est le résultat de ce qui s'apparente à une "somme" (cf. citations (10) à (12) pour la phrase, note 6 (I: 92) pour le système). Ce "sens" ne peut donc être une sorte de lieu géométrique, de moyenne, de contenu sociologiquement stabilisé issu des données discursives; considérons alors que cette "totalité" désigne l'organisation de leurs formes en un système de "rapports". Dès lors entre les désignations accumulées et $l e$ "sens" il y a rupture, bond qualitatif. Rupture avec la partie du discours non traitée par la linguistique d'E.B.: le pur pragmatique et "l'intenté" subjectif, bien qu'ils ne soient pas sans effet sur le sens et sur l'évolution du système; mais surtout rupture de par l'arbitraire de la clôture du corpus, lequel, généralement écrit, est prélevé dans une situation sociolinguistique de diglossie opposant variété (voire souvent langue) orale et écrite. Mais encore rupture avec l'intuition commune laquelle ne fournit pas un critère absolu : les notions partagées par la "communauté linguistique", celles qui constituent dans les dictionnaires "un réseau de correspondances acquises" (1969, I : 9), sont susceptibles d'être invalidées (II : 272 et $s q$, I : 295, etc.). Le saut 
qualitatif est sensible lorsque la signification apparaît une là où existait la multiplicité "de données peu homogènes engagées dans un développement imprévisible" (1969, I : 10). Le geste établissant la signification touche à la fonction instituante de la langue : Benveniste révèle un rapport nécessaire ("biunivoque" écrit Montaut, 1992: 124) entre telles unités sémiotiques et le sémantique, ceci alors qu'"une langue est radicalement impuissante à se défendre contre les facteurs qui déplacent d'instant en instant le rapport du signifié et du signifiant" (Saussure cité par E.B., I : 53, 1939)21. Le "rapport de signification" manifeste bien cette nécessité, il est l'effet de l'asservissement du sémantique par le sémiotique. Ce dernier apparaît irréductible: deux termes associés à une notion semblable ne sont pas permutables, ainsi en est-il, nous l'avons vu, de civitas et de polis, qui ne sont pas substituables étant donné les relations inverses qui les associent respectivement à civis et à politès ; ce rapport de signification (ici, entre quatre signes) engage pour chacun une différence notionnelle. La mise en évidence d'un système lexical détermine un principe sémantique stable, sorte d'idéal du sens des signes mis en relation. Les emplois sont situables par rapport à ce principe; celui-ci les rend-il entièrement prévisibles? Autrement dit ce geste suture-t-il le hiatus dans la langue? L'établissement du rapport de signification rétablit les droits du sémiotique sur le sémantique qui a fourni cette fois les matériaux : il y a bien sémiotisation du sémantique, la signification est transformation de désignations. Ce geste réalise le second temps du capitonnage nécessité par le clivage de la langue. L'interstice est maintenu. D'une part le micro-système du "rapport" lexical constitue un paradigme, la "signification" qu'il construit est principielle et possède d'évidence une "valeur générique et conceptuelle". D'autre part le procès de "sémantisation de la langue", qui lui est lié, reste hétérogène: il réalise la désignation du référent à l'aide d'un événement chaque fois "unique", "évanouissant" : la phrase, et produit un sens "particulier, ou occasionnel" ; c'est du reste dans la langue "en emploi et en action" que les termes sont engagés dans une erre au "développement imprévisible"22.

Par ce repliement, les temps logiques de la constitution du sens et de la signification se bouclent, du pur sémiotique et retour par le sémantique, sur un sémiotique remanié.

Les deux ordres lus chez E.B. s'enchaînent selon 3 phases logiques dont la première est reconstruite, leur consécution vient de ce qu'un même matériel remplit une fonction double, une dans chaque phase. La première, SIGNIFIER, vaut pour la reconnaissance sémiotique de ce qui se donne comme amorce et qui sera validée rétroactivement par la scansion qui établit l'unité phrase, dotée

21 Glissement vérifié par la variété des désignations. "Le rapport du signifié et du signifiant paraît toujours fluide, toujours prêt à se défaire" (Lacan, $1981: 297$ ).

22 Donnons cet exemple tiré d'un argot de jeunes des cités: "boulet" vaut pour "fille", mais laquelle ? Celle qu'on traîne ? Le "pot à tabac" ? Pas du tout, il s'agit d'une "meuf canon". 


\section{Marc Derycke}

d'une référence (premier temps de la phase II) ; la séquence sémiotique à quoi est associée la référence constitue le maillon enchaînant I à II (I.1 = II.1, soit la "scansion", ou constitution de la phrase). La seconde phase, COMMUNIQUER, est celle du sémantique dont la phrase est l'expression par excellence, son second temps est celui de la délimitation de ses constituants et l'identification de leur désignation (II.2 = III.2). Le mot, en tant que constituant de la phrase, est le maillon qui permet d'enchaîner avec la phase III, que je nomme INSTITUER, phase de l'instauration du principe sémantique (le rapport de signification) à partir d'un rapport lexical. Elle vient d'un repliement de la seconde phase sur la première et forme un sémiotique "toujours menacé".

I. SIGNIFIER I.0. séquence sémiotique (amorce)

\section{I.1. scansion}

II. COMMUNIQUER II.1. phrase : prédication -> référence (I.1 = II.1)

\section{INSTITUER}

\section{II.2. identification des éléments de $P$}

III.2. mot avec désignation (II.2 = III.2)

\section{III.3. association termes + notions (rapport de signification)}

Les deux premières phases scandent l'actualisation de la langue dans son procès de sémantisation (discours), la troisième réalise sa formalisation et sa virtualisation dans un procès de sémiotisation; le sémiotique, pris comme forme pure, est à la fois origine et aboutissement de ces phases.

Les concepts descriptifs du métalangage doivent être lus selon ce mouvement en boucle, lequel peut se répéter : leur univocité tient aux temps comptés sur la spirale. Ainsi, outre la langue en emploi ou comme système, dans la citation (10), phrase témoigne du repliement de II.1 sur I. De même en (9) la "coïncidence" "pratique" du mot avec le signe est consistante, elle est le produit du raboutage du sémantique II.2, ("élément syntagmatique"), avec le sémiotique dont I est la manifestation ${ }^{23}$.

\footnotetext{
23 Dans cet article ("Niveaux..."), "coïncidence" présuppose distinction, du reste E.B. réclame ici l'indulgence pour la "commodité" de l'"analyse". De plus la distinctivité du signe relève aussi de sa capacité d'intégration au "niveau" de la phrase en ce qu'il y remplit en tant qu'élément une fonction propositionnelle, ce qui engage son sens (sémiotique) par sa distribution, et non point le sémantique, lequel est suspendu à la fonction prédicative de la phrase en tant que totalité dont dépend la référence. Contrairement au point de vue de de Vogüe, il me semble donc que, pour la distinction mot / signe les articles postérieurs constituent non pas un revirement mais plutôt un approfondissement : "Niveaux..." rend compte du nouage du sémiotique au sémantique, "La forme et le sens..." réalise le nouage inverse.
} 
La sentence qui clôt les "Niveaux d'analyse..." rend compte du mouvement en boucle sur trois phases: "C'est dans le discours, actualisé en phrases (I et II, indissociables temporellement mais disjoints, n.d.r.), que la langue se forme et se configure (phase III, $i d$.). Là commence le langage (en I et II, id.) (...) : nihil est in lingua quod non prius fuerit in oratione" (I : 131). La greffe du capitonnage permet donc d'introduire un principe de cohérence dans un métalangage apparemment "brouillé" par le clivage de la langue.

La résultante de ces trois phases tient en cette fonction supplémentaire qu'E.B. lui assigne: "bien avant de servir à communiquer, le langage sert à vivre" (II : 217). Ce qui ramène au départ (I), ouvrant sur le poétique en gésine :

"au fondement de tout, il y a le pouvoir signifiant de la langue, qui passe bien avant celui de dire quelque chose (...) attribut que nous mettons au coeur le plus profond du langage: oute legei, oute kriptei "Il ne dit, ni ne cache", alla sémainei "mais il signifie". (II : 229)"24

\section{Bibliographie}

ARRIVE, M., 1994, Langage et psychanalyse, linguistique et inconscient - Freud, Saussure, Pichon, Lacan, Paris, P.U.F..

BENVENISTE, E., 1966 et 1974, Problèmes de linguistique générale I et II, Paris, Gallimard.

- 1969, Le vocabulaire des institutions indo-européennes I, Paris, Minuit.

CULIOLI, A., 1984, "Théorie du langage et théorie des langues", L'information grammaticale, "E. Benveniste aujourd'hui" tome I (Actes du Colloque international du CNRS, Université F. Rabelais, Tours 1983), Paris.

DERYCKE, M., 1990, Lecture(s) : comment le sens vient aux mots, doctorat dirigé par F. Regnault, Département de Psychanalyse, Saint-Denis, Université Paris VIII.

- 1995, "Le clivage du signe selon E. Benveniste", Langage et société n 70, Paris, Maison des Sciences de l'Homme.

de VOGUE, S., 1992, "Culioli après Benveniste : énonciation, langage, intégration", Linx n² 26, Nanterre, Université Paris X.

24 Raboutons ces deux aphorismes qui clôturent ces deux articles, on obtient une contradiction liée à celle relevée en 1939: " entre le signe et l'objet (...) la relation en même temps se modifie ("menacée" in oratione, n.d.r.) et reste immuable (dans le système "restauré", idem)" ; la seconde contradiction est cause de la première. 


\section{Marc Derycke}

ECO, U., 1985, Lector in fabula, Paris, Grasset et Fasquelle.

FREGE, G., 1971, Ecrits logiques et philosophiques, trad. C. Imbert, Paris, Seuil.

FREUD, S., 1930, Le mot d'esprit et ses rapports avec l'inconscient, Paris, Gallimard.

LACAN, J., 1966, Ecrits, Paris, Seuil.

- 1981, Le séminaire, Livre III : "Les psychoses", Paris, Seuil.

MONTAUT, A., 1992, "La méthode de Benveniste dans ses travaux comparatistes : son discours et son sujet", Linx $\mathrm{n}^{\circ} 26$, Nanterre, Université Paris X.

NORMAND, C., 1986, "Les termes de l'énonciation de Benveniste", Histoire, épistémologie, langage VIII-2, Saint-Denis, P.U.V.

- 1989, "Constitution de la sémiologie chez Benveniste", Histoire, épistémologie, langage XI-2, SaintDenis, P.U.V.

- 1992, "Benveniste : linguistique saussurienne et signification", Linx $n^{\circ} 26$, Nanterre, Université Paris X.

TAMBA-MECZ, I., 1984, "A propos de la distinction entre "sémiotique" et "sémantique" chez E. Benveniste", L'information grammaticale, "E. Benveniste aujourd'hui" tome I (Actes du Colloque international du CNRS, Université F. Rabelais, Tours 1983), Paris. 